\title{
Antisense Technology
}

\author{
Mahnoor Patel \\ Department of Biotechnology, Veer Narmad South Gujarat University, Surat 395007, Gujarat, India. \\ Email: patelmahnoor786@gmail.com
}

\begin{abstract}
Diseases are often connected on the expression of some disease causing gene which is important to produce that "Protein." If the expression of this gene can be disputed then the disease can be cured. Antisense technology is a method of disputing the production of protein. It may be used to design some therapeutics for diseases in whose pathology is the production of protein plays a major role. Antisense technology is important tool in the inhibition of that particular gene expression. The principle behind it, is that the antisense nucleic acid sequence base pairs with its complementary sense RNA strand is inserted and prevents the RNA from translated into protein. The complementary nucleic acid sequence is either complementary synthetic oligonucleotide, often oligodeoxy ribonucleotide, or longer antisense RNA sequence.
\end{abstract}

\section{Introduction}

Diseases are mostly related to the overexpression of some disease causing gene. Gene can be translated to produce the final product called protein. If the protein is able to cause disease, then this type of disease can be treated with the use of antisense technology. This technology can be used to inhibit the protein. The pathogenic organisms have the capacity to incorporate their gene within the human genome, then the gene can be translated by using the host machinery.

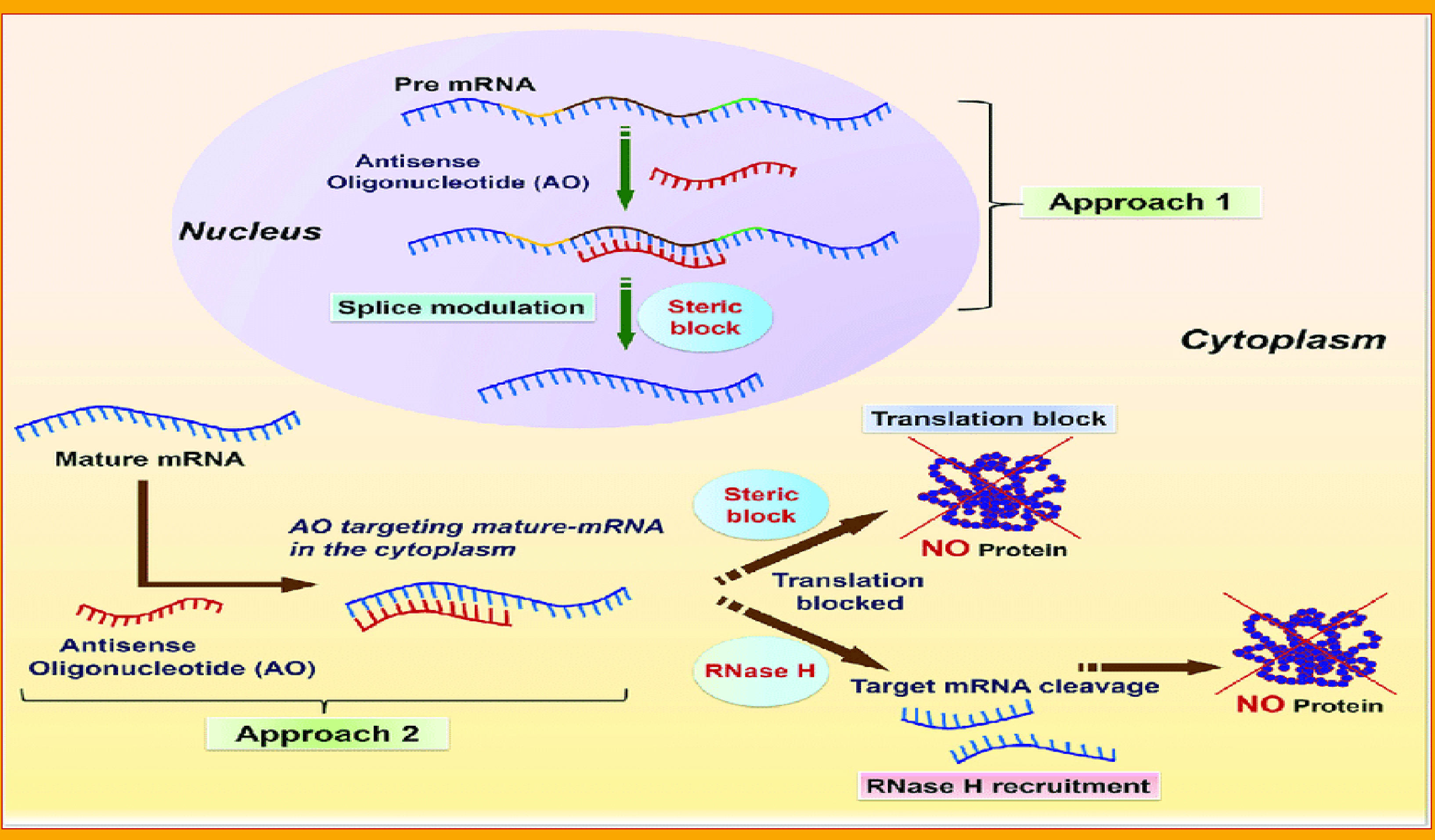

\section{Mechanism}

A sense strand is a $5^{\prime}$ to $3^{\prime}$ mRNA molecule of DNA or RNA. The complementary or mirror strand, it is called an antisense strand. The therapeutic objective of this technology is to block the disease causing protein. This can be achieved by the addition of the antisense strand of the sense strand. After the formation of mRNA, the antisense complex can be formed. This complex cannot be transfer for longer time. This complex will be degraded.

\section{Mechanism of Antisense Technology}

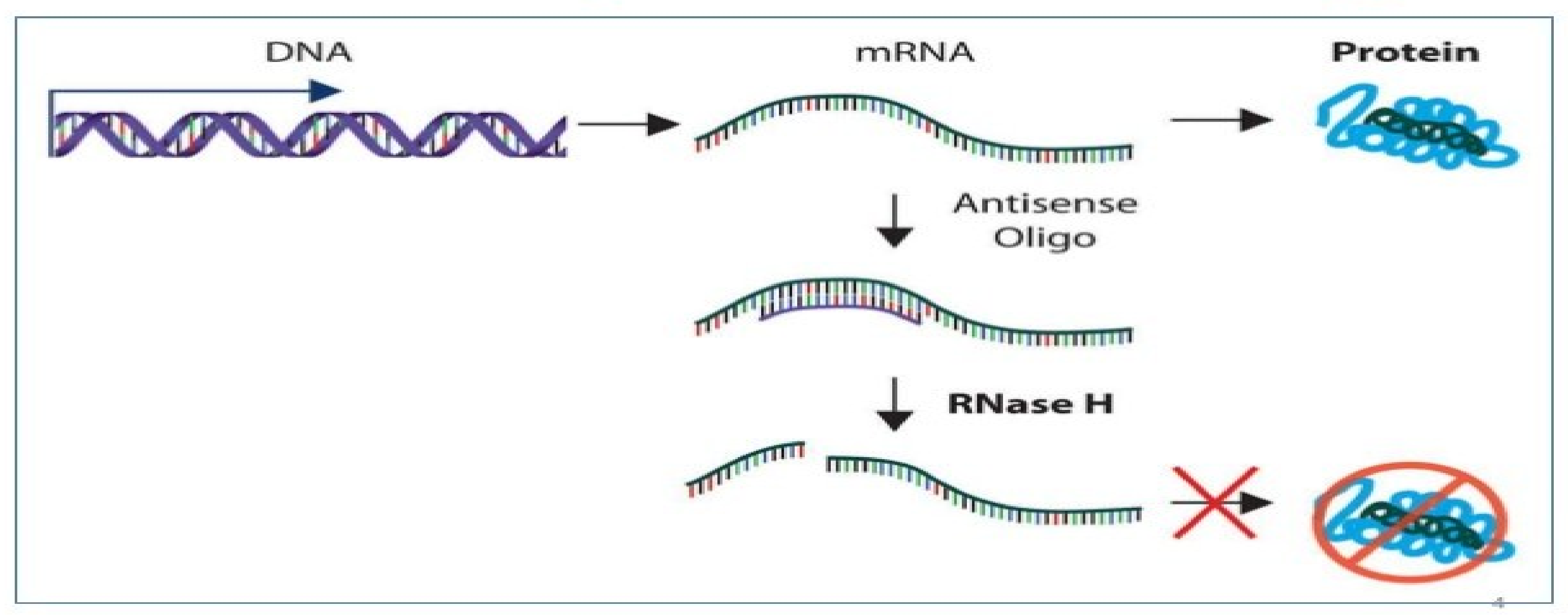

\section{Insertion of an Antisense RNA into Cells}

1. Endocytosis: Receptor mediated endocytosis. Drawback is time consuming, unreliable and inefficient.

2. Micro Injection: The sample can be injected directly, yield is very high, but great precision is needed. Work is very tedious.

3. Liposome Encapsulation: Cationic phospholipid bilayer will surround the nucleotide sequence. The resulting liposome can be attached to the cell and allow antisense RNA to enter into the cell, but this method is very expensive.

4. Electroporation: The antisense molecule should transverse the cell membrane offer a shock is applied to the cell.

5. Antisense PG Gene: PG enzyme can be able to breakdown the pectin present in the cell wall and it gives the firmness to the tomato. The PG mRNA present in the tomato can be blocked by the researcher to slow down the softening process of tomato. It is used in flavr savr tomato. This type of tomato can be store for a longer time.

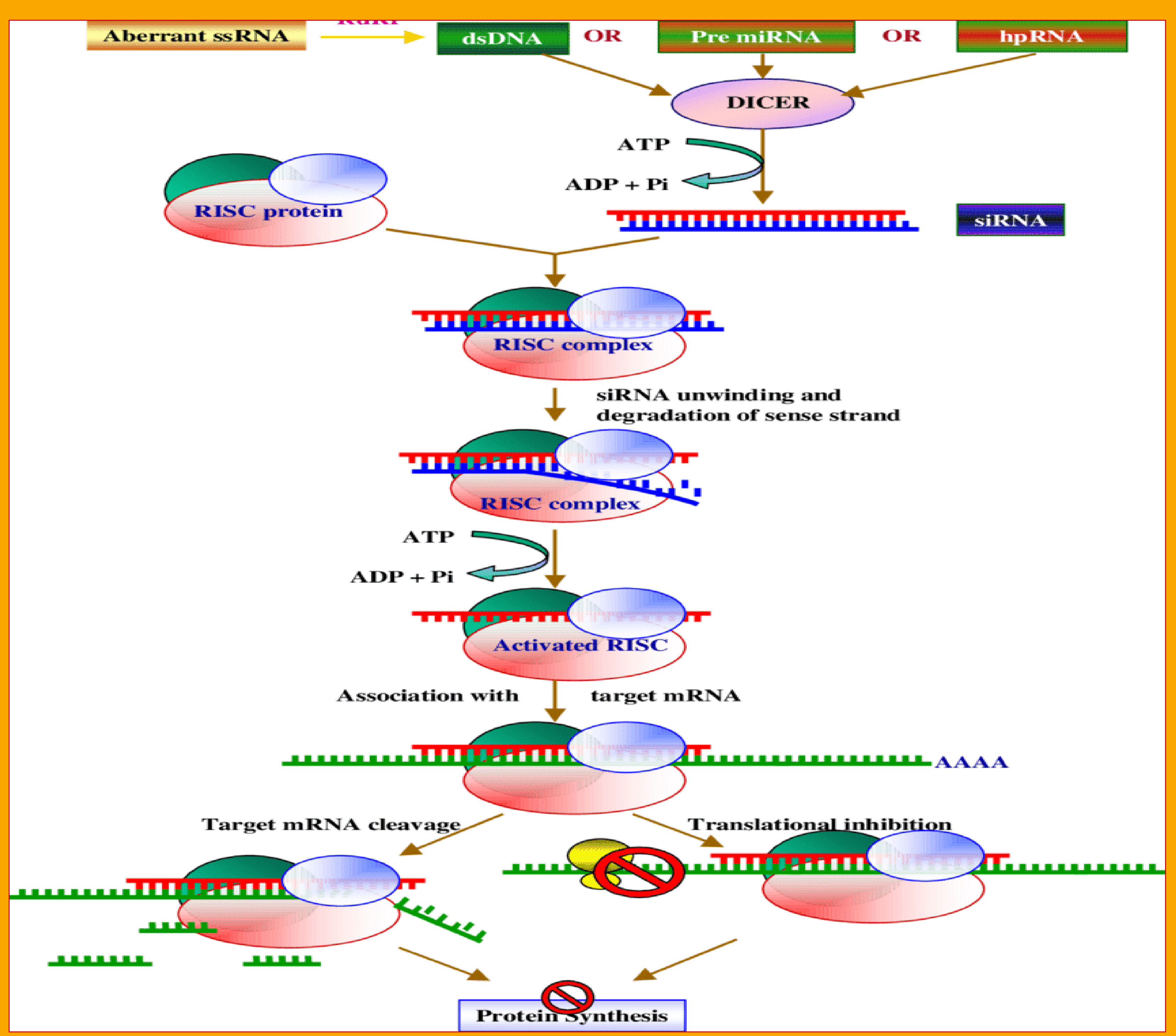

\section{Principle}

The principle in this technology is that an antisense nucleic acids base pairs with its complementary sense RNA strand and prevents it from being translated into a protein. This type of complementary sequence can be either a synthetic oligonucleotides or oligodeoxy ribonucleotides of less than 30 nucleotides or longer than 30 nucleotides sequences. Antisense strand is totally an opposite strand of the sense mRNA strand.
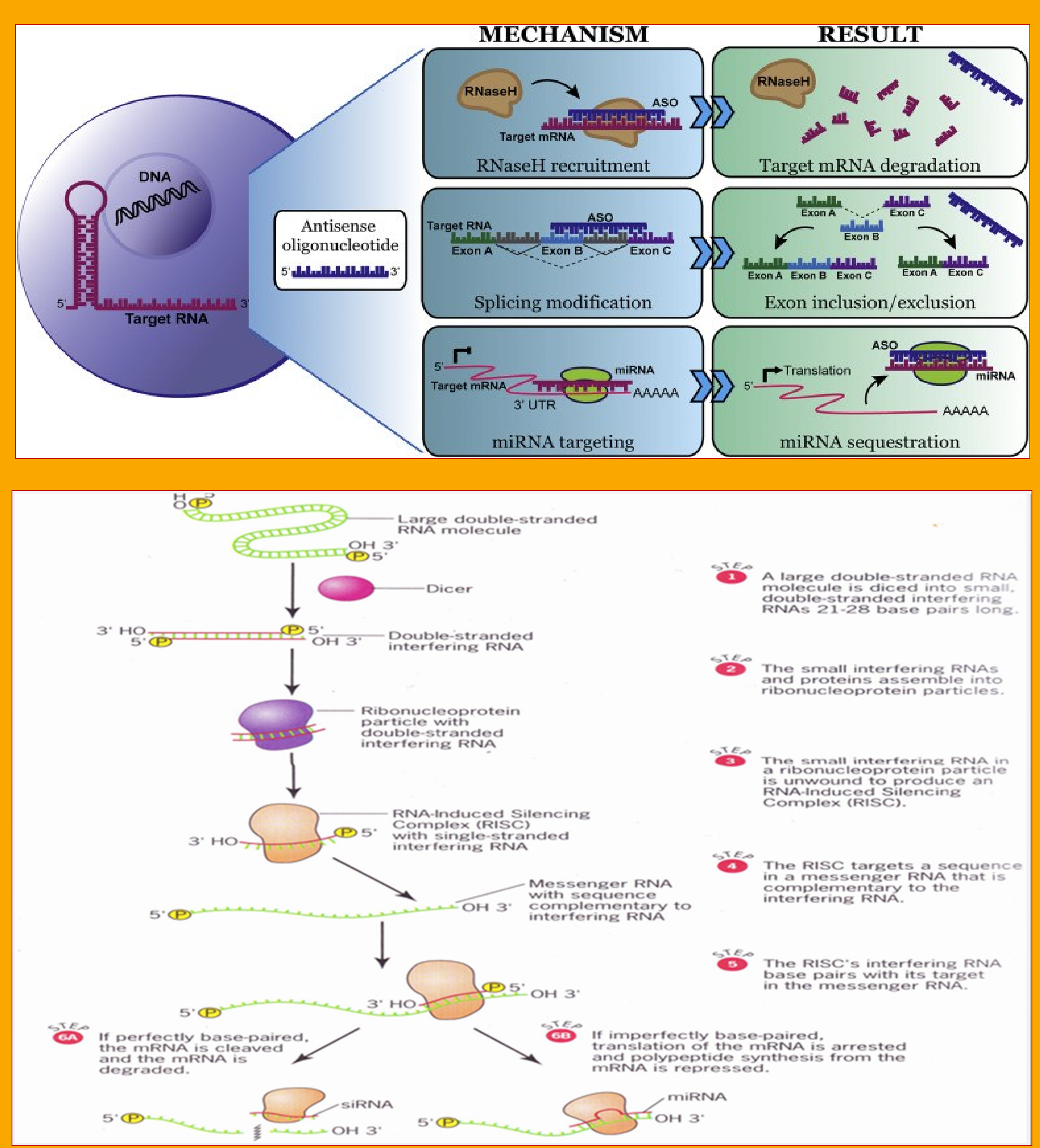